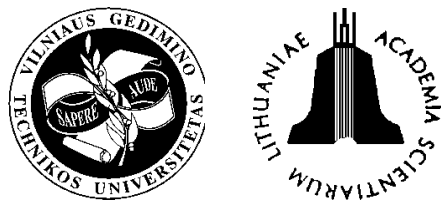

ISSN 1648-4142 TRANSPORT

http:/www.vtu.lt/english/editions

\title{
REDUCTION OF VIBRATIONS IN ELECTROMECHANICAL DRIVE SYSTEMS WITH VECTOR CONTROL
}

\author{
Paweł Bachorz, Eugeniusz Świtoński, Arkadiusz Mężyk \\ Dept of Applied Mechanics, Silesian University of Technology, Konarskiego 18A, 44-100 Gliwice, Poland. \\ E-mail: Arkadiusz.Mezyk@polsl.pl \\ Received 2004-09-01; accepted 2004-10-07

\begin{abstract}
This paper presents the issues of modelling electromechanical drive systems in a mechatronic approach. The effect of a vector control system on the dynamics of the system is shown. The optimisation of the system in order to minimise vibration amplitudes and amplitudes of forces in selected kinematic pairs of a high power drive system was performed with the use of genetic algorithms. Design variables of the optimisation process comprised the structural parameters of the mechanical part as well as the settings of PI controllers of the vector control system. The selected results of numerical computations are presented.
\end{abstract}

Keywords: optimization, genetic algorithm, dynamic, electromechanical drive systems, vector control.

\section{Introduction}

Contemporary working environment of high power machines and electromechanical devices sets constantly rising requirements on their dynamic properties. Complex operating tasks have to be carried out and the required working tool trajectories have to be maintained under dynamically variable loads. The complex structure of the device and dynamic changes of load generate vibrations in the system and, subsequently, noise, which is a nuisance to the operators of the device. Vibrations cause initiation of fracture growth, which leads to failures and destruction of the structure. Under market economy conditions the rate of energy consumption by these systems is also important, and it is influenced not only by the dynamics and the structure of the system, but also by the manner of its control. These elements can be optimised already at the stage of design. These problems can efficiently be analysed only by means of mathematical modelling [1, 2]. Simulation tests enable a more thorough study of phenomena occurring in a given object and are usually less expensive than experimental tests. They also allow the analysis of failure states which is impossible in the case of experimental tests of real objects, as such tests could pose hazard to human life or cause the destruction of costly equipment.

The authors of this paper decided to study the efficiency of the use of vector control in an induction motor to reduce dynamic loads in kinematic pairs of an electromechanical system. Possibilities of tuning the mechanical and electrical part and the control system have been analysed in such a manner as to arrive at the lowest possible amplitudes of dynamic interaction in kinematic pairs of the system. An electromechanical model with a control system was applied because of the complexity of dynamic phenomena. Proper selection of the selected design features was made with the use of a genetic algorithm. This enabled to avoid the need to differentiate the objective function.

\section{Model of an electromechanical drive system}

In electromechanical systems, both the electrical and the mechanical part of the drive are a dynamic system. These parts are intercoupled. It is therefore necessary, when analysing dynamic phenomena, to apply a model that enables electromechanical coupling. Vector drives, which have been developed and improved in recent years, enable accurate speed control and full control of the torque of a squirrel-cage motor. The solutions applied vary in the methods of affecting the speed and torque and in the manner of evaluating the internal state of the motor. The most widespread solution is the Field Oriented Control. Separate control of the rotor current, which is responsible for the torque, and of the magnetising current, which generates flux, enables these drives to attain operating parameters comparable to those of direct 
current drives. The frequency converter can therefore control the speed and the torque independent of each other.

Tests were carried out for an electromechanical drive of the head of a mining longwall shearer (Fig 1). The physical model of that drive with 119 degrees of freedom is shown in Fig 2. The system comprised:

- an electric motor driving the system (three-phase induction motor for mining equipment, type SG 4562 L4),

- a kinematic chain for power transmission, con-

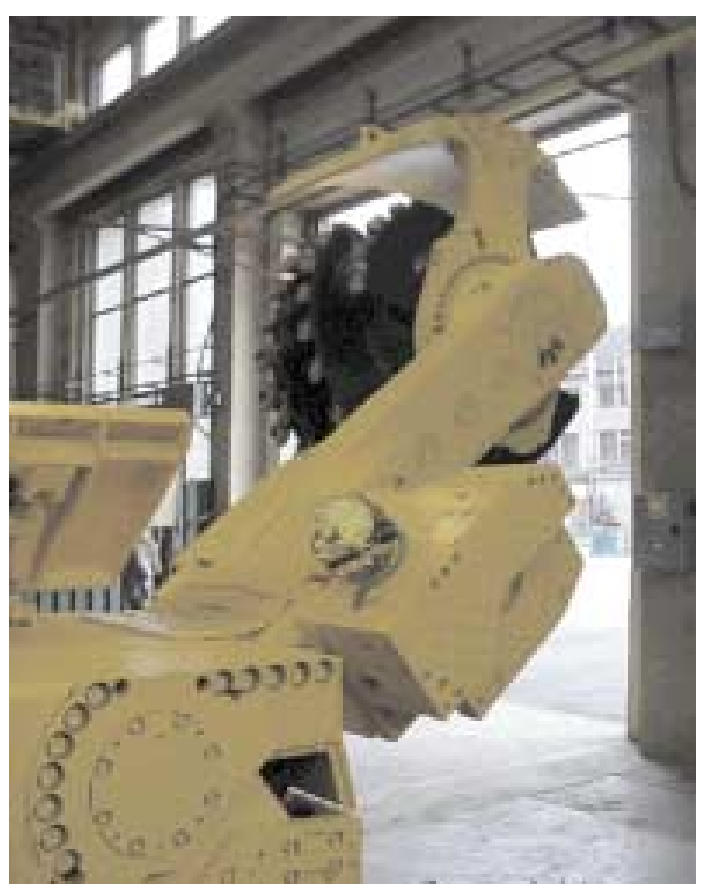

Fig 1. System under consideration sisting of several stages of toothed gear train and a planetary gear with overall transmission ratio between the rotor of the motor and the shearing tool $\mathrm{i}=38,3$,

- a shearing tool with a diameter of $1500 \mathrm{~mm}$.

The model of the mechanical part is described by a system of ordinary differential equations:

$$
\mathbf{M} \ddot{\mathbf{q}}+\mathbf{C} \dot{\mathbf{q}}+\mathbf{K q}=\mathbf{Q},
$$

where $\mathbf{M}$ - matrix of inertia, $\mathbf{C}$ - matrix of damping, $\mathbf{K}$ - matrix of stiffness, $\mathbf{Q}$ - vector of generalised forces, $\mathbf{q}-$ vector of generalised displacements.

The electric motor is described by means of a polyharmonic model [3]:

$$
\begin{aligned}
& {\left[\begin{array}{c}
\mathbf{u}_{\mathbf{s}} \\
\mathbf{0}
\end{array}\right]=\left[\begin{array}{cc}
\mathbf{R}_{\mathrm{s}} & \mathbf{0} \\
\mathbf{0} & \mathbf{R}_{\mathbf{r}}
\end{array}\right] \cdot\left[\begin{array}{c}
\mathbf{i}_{\mathbf{s}} \\
\mathbf{i}_{\mathbf{r}}
\end{array}\right]+\left[\begin{array}{cc}
\mathbf{L}_{\text {rs }} & \mathbf{0} \\
\mathbf{0} & \mathbf{L}_{\mathbf{r r}}
\end{array}\right] \frac{d}{d t}\left[\begin{array}{l}
\mathbf{i}_{\mathbf{s}} \\
i_{\mathbf{r}}
\end{array}\right]+\frac{d}{d t}\left\{\left[\begin{array}{cc}
\mathbf{L}_{\mathrm{ss}} & \mathbf{L}_{\mathbf{s r}}(\varphi) \\
\mathbf{L}_{\mathrm{sr}}^{\mathrm{T}}(\varphi) & \mathbf{L}_{\mathbf{r r}}
\end{array}\right] \cdot\left[\begin{array}{l}
\mathbf{i}_{\mathbf{s}} \\
\mathbf{i}_{\mathbf{r}}
\end{array}\right]\right\}} \\
& \frac{d}{d t} \omega=\frac{1}{J}\left(\mathbf{i}_{\mathbf{s}}^{\mathbf{T}} \cdot \frac{d}{d \varphi} \mathbf{L}_{\mathbf{s r}}(\varphi) \cdot \mathbf{i}_{\mathbf{r}}-M_{o}\right), \\
& \frac{d \varphi}{d t}=\omega,
\end{aligned}
$$

where $\mathbf{u}_{\mathrm{s}}-$ vector of stator voltages, $\mathbf{i}_{\mathrm{s}}, \mathbf{i}_{\mathrm{r}}-$ vector of stator and rotor currents, $\mathbf{R}_{\mathrm{s}}, \mathbf{R}_{\mathrm{r}}$ - matrix of stator and rotor resistances, $\mathbf{L}_{\mathrm{ss}}, \mathbf{L}_{\mathrm{rr}}-$ matrix of stator and rotor self-inductances, $\mathbf{L}_{\mathbf{s r}}$ - matrix of mutual inductances stator-rotor.

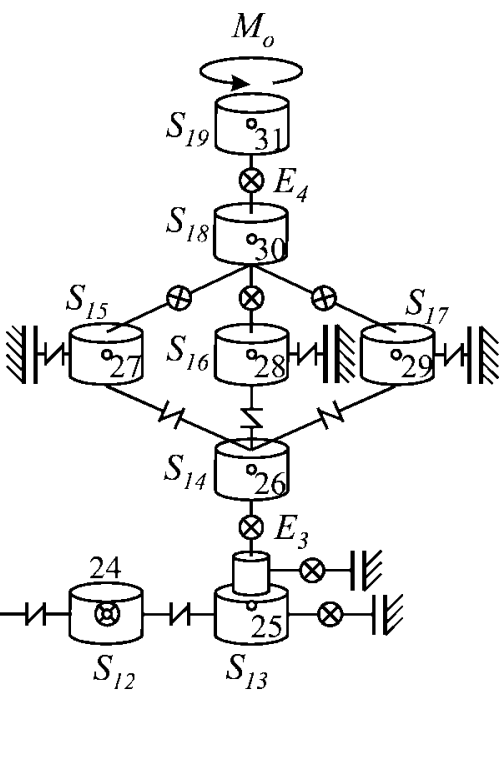

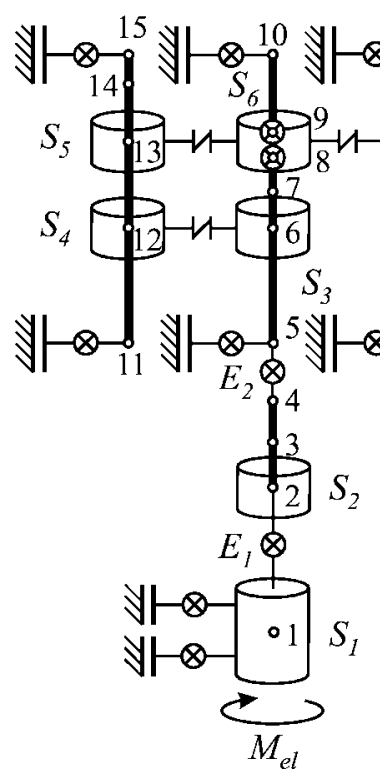

Fig 2. Model of transvere-torsional vibration 


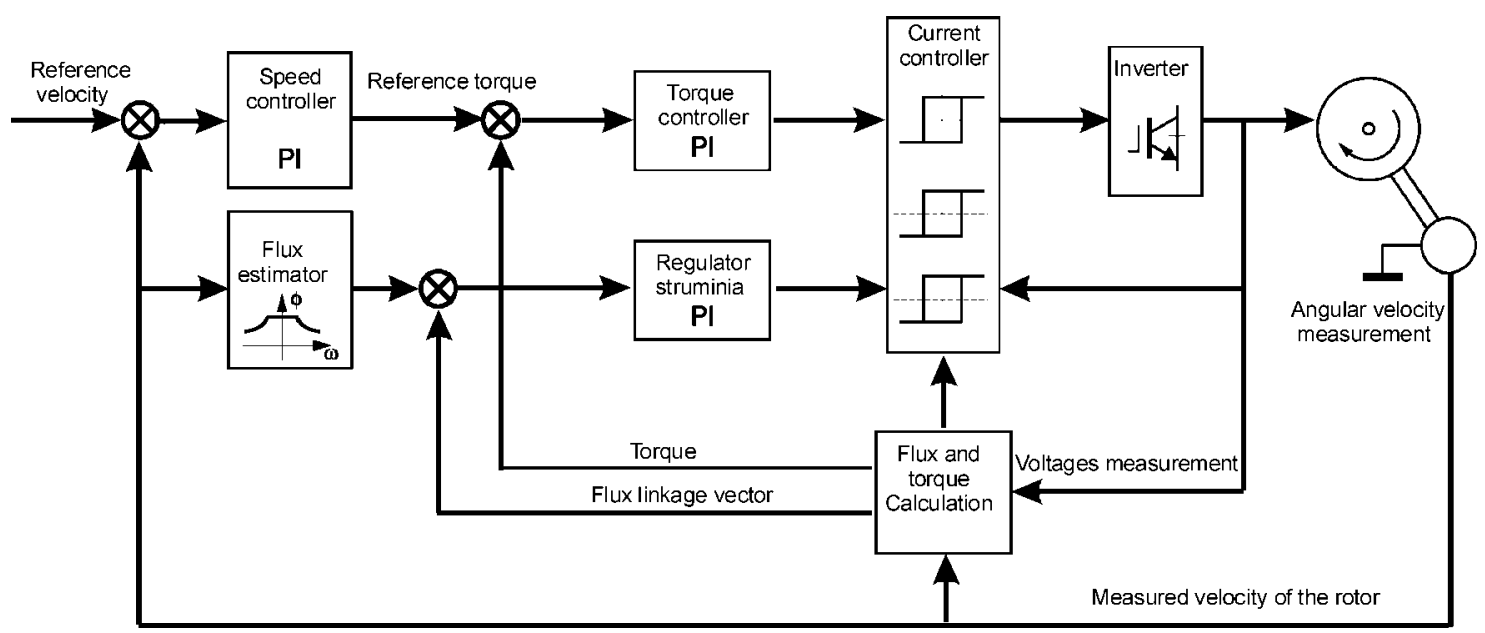

Fig 3. Signal flow graph of a vector control unit [4]

A model of the vector control system of the motor (Fig 3) with feedback from the mechanical part was also applied in the tests. It enables accurate control of speed and torque while retaining high dynamics of the drive [4]. The use of vector control system improved the dynamics of the drive considerably, which is reflected in Fig 4 showing the start-up of the motor.

\section{Minimisation of vibrations in an electromechani- cal drive system}

Minimisation of vibrations in the drive system of the head of a mining shearer, treated as a mechatronic system, was performed with the use of genetic algorithms. The reduction of the amplitude of vibrations was attained by optimising the objective function that describes the electromagnetic moment of the motor in the following form:

$$
\psi=M_{e l}^{2} .
$$

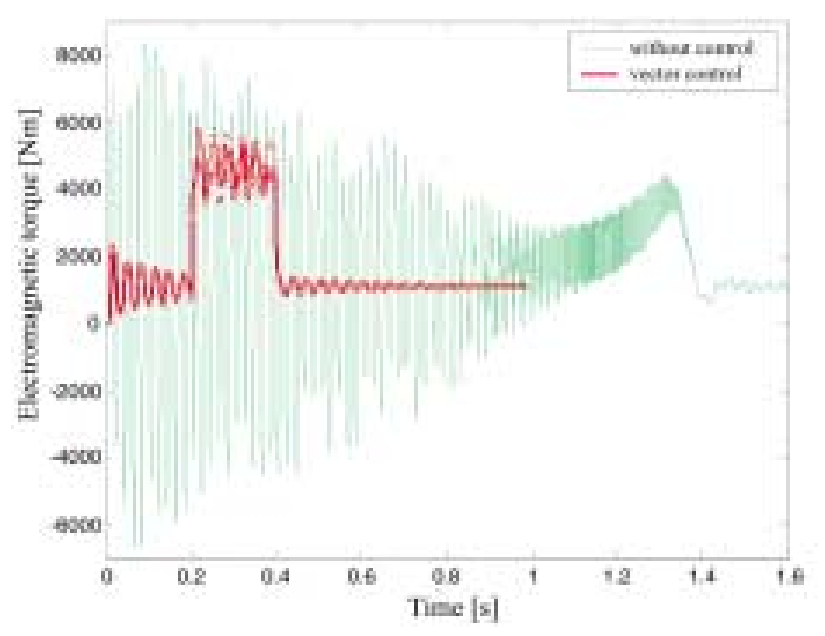

Fig 4. Electromagnetic torque of AC motor
Genetic algorithms were employed to solve the optimising task. The set of decision variables comprised the settings of controllers of the control system and selected structural parameters of the mechanical part of the system. Three variants of optimisation were implemented. In the first variant the only decision variables were the structural parameters of the mechanical part. In the second variant the decision variables were the settings of the controllers in the control system, whereas in the third variant they included both the settings of controllers as well as the structural parameters.

\section{Numerical computations}

The simulation of the drive system operation was performed for four variants of computations:

- variant 1 (a) - system prior to optimisation,

- variant 2 (b) - system after optimisation of the settings of vector control PID controllers,

- variant 3 (c)- system after optimisation of structural parameters of the mechanical part,

- variant $4(\mathrm{~d})$ - system after optimisation of the settings of vector control PID controllers and of structural parameters of the mechanical part.

The results obtained after the optimisation of the system under resistance torque corresponding to the rated torque of the motor are shown in Fig 5. This figure illustrates the changes in the electromagnetic moment of the motor obtained in all computation variants. As it can be seen optimisation has reduced the amplitude of oscillations of the electromagnetic moment.

The lowest oscillations of the electromagnetic moment are observed after the optimisation of both the settings of vector control PID controllers and of structural parameters of the mechanical part. The 


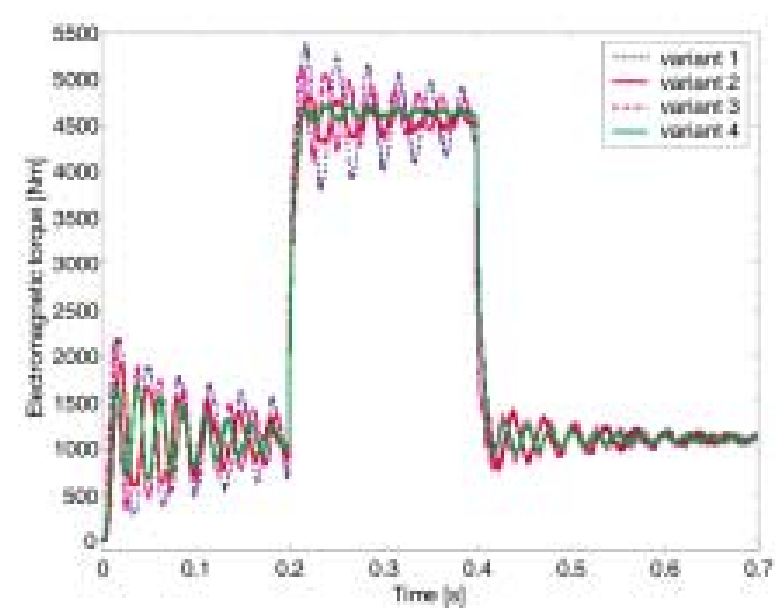

Fig 5. Electromagnetic torque of $\mathrm{AC}$ motor with the vector control unit

highest oscillations were obtained after the optimisation of only the structural parameters of the mechanical part. The amplitude of the electromagnetic moment was considerably reduced both in the phase of system start-up and during steady operation.

Figure 6 shows changes of the moment in a selected kinematic pair for various computation variants. This graph also shows a significant reduction of the amplitude of moment oscillations in the system after the optimisation of both the settings of vector control PID controllers and of structural parameters of the mechanical part (d) in relation to the system prior to optimisation.

Figure 7 shows changes of power absorbed by the electric motor during a steady operation with the same operating parameters for various computation variants. The reduction of the amplitude of oscillations and of absorbed power can also be seen here. The largest reduction is observed after the optimisation of both the settings of vector control PID controllers and of structural parameters of the mechanical part.

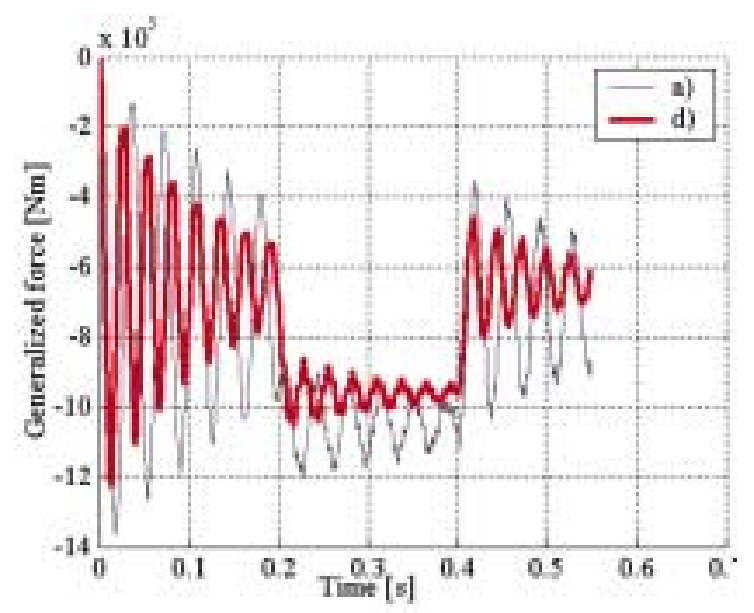

Fig 6. Dynamic torque in a selected kinematic pair

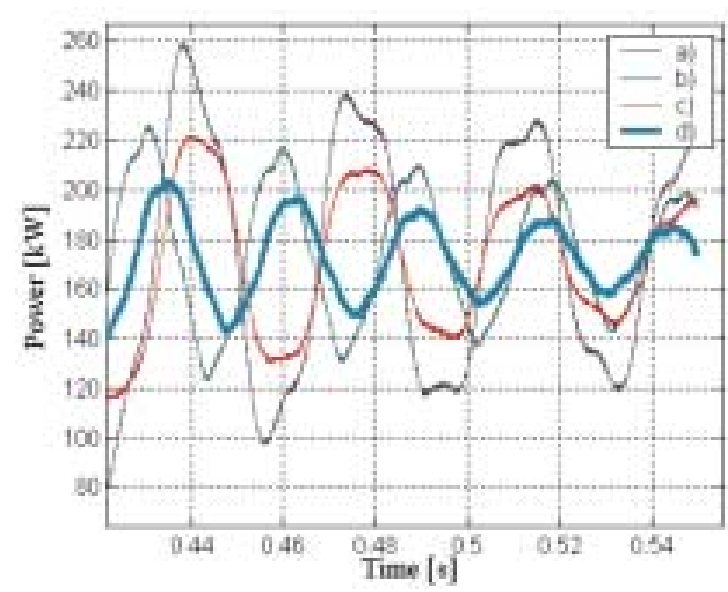

Fig 7. Power of the motor vs. time

\section{Conclusions}

This paper presented a method of modelling drive systems of high power machines. Each developed model consisted of a mechanical part and electrical part coupled by means of angular velocity of the drive motor and by electromechanical moment. These models were applied to minimise the vibrations generated in the system. The optimisation of the systems in a mechatronic approach was performed with the use of genetic algorithms. The effect of selected parameters on the improvement of dynamic properties of drive systems was shown.

Marked improvement was achieved after applying vector control and optimising its settings. The results obtained proved the usefulness of vector control in such systems. It would be advisable to carry out experimental tests in order to verify the results obtained for a controlled system. However, it was not possible to run such experiments because of the specificity of system operation and the fact that systems of this class have not been equipped with vector control systems yet.

\section{References}

1. Bachorz, P. Minimization of the vibrations of electromechanical drive systems. PhD Thesis Silesian Technical University, Faculty of Mechanical Engineering. Gliwice, 2003.

2. Mężyk A. Modelling and optimization of transmission systems with an asynchronous motor. Journal of Theoretical and Applied Mechanics, 1(41), 2003, p. 169-198.

3. Trawiński, T. Effect of parasitic electromagnetic moments on dynamic properties of induction motors supplied by inverters. PhD Thesis, Silesian Technical University, Faculty of Electrical Engineering, Gliwice, 2000.

4. Kuś, W. Vector control in AC motors. Elektronika Praktyczna, 9/2000, p. 51-56. 